\title{
Data Mining Based Approach for Evaluation of EEG Signals for Epilepsy Detection
}

\author{
AYMAN M. MANSOUR \\ Department of Communication, Electronics and Computer Engineering, \\ Tafila Technical University, Tafila, 66110, JORDAN \\ MOHAMMAD A OBEIDAT \\ Department of Power and Mechatronics Engineering, \\ Tafila Technical University, Tafila, 66110, JORDAN \\ MURAD AL-AQTASH \\ Department of Communication, Electronics and Computer Engineering, \\ Tafila Technical University, Tafila, 66110, JORDAN
}

\begin{abstract}
The objective of this proposed research is to come up with a general methodology for classification of time series events, and to apply that methodology to the analysis of physiological signals recorded from epileptic patients for seizure analysis depending on EEG signal. In contrast to previous works, this research considered an alternative formulation of seizure analysis as a detection problem. This approach offers a good treatment of seizure detection.
\end{abstract}

Key-Words: - Epilepsy, Standard Deviation, Entropy, Power Spectrum density, Discrete Time Wavelet Transform.

Received: June 21, 2019. Revised: April 13, 2020. Accepted: April 30, 2020. Published: May 22, 2020.

\section{Introduction}

The electroencephalogram (EEG) signals have long been recorded and studied as potentially of the electric activity of the brain. It has stood the test of time because it monitors physiological functions as these changes with time and is reactive to many factors. EEG reflect brain electrical activity with millisecond temporal resolution, and are the most direct correlate of on-line brain processing obtainable non-invasively.

The electrical activity of active nerve cells in the brain produces currents spreading through the head. These currents also reach the scalp surface, and resulting voltage differences on the scalp can be recorded as the electroencephalogram (EEG). The (EEG) signal contains-among others-four main spectral components. The most important one is the $\alpha$ wave, having the frequency between $8-12 \mathrm{~Hz}$. Higher frequency components (over $12 \mathrm{~Hz}$ ) are denoted by $\beta$, these waves have lower magnitudes. Lower frequency components $\delta$ (under $4 \mathrm{~Hz}$ ) and $\theta$ (between $4-8 \mathrm{~Hz}$ ) are also present, both having low magnitudes in normal cases [2].

The clinical interests in (EEG) are; for example, sleep pattern analysis, cognitive tasks registration, seizure and epilepsy detection, and other states of the brain, both normal and patho- physiological. Epilepsy is the second most prevalent neurological disorder in humans after stroke. It is characterized by recurring seizures in which abnormal electrical activity in the brain causes altered perception or behavior. Well-known causes of epilepsy may include: genetic disorders, traumatic brain injury, metabolic disturbances, alcohol or drug abuse, brain tumor, stroke, infection, and cortical malformations (dysplasia).

A seizure is a disturbance characterized by changes in neuronal electrochemical activity that results in abnormal synchronous discharges in a large cell population, giving rise to clinical symptoms and signs. These are some of the seizure patterns that one sees on EEG: 1) Absence seizures have the characteristic $3 \mathrm{hz}$ spike and wave discharges. 2)Juvenile myoclonic epilepsy has a $4 \mathrm{~Hz}$ spike and wave discharge pattern 3)Infantile spasms show the ominous pattern of hypsarrhythmia 4) Lennox Gastaut shows 1-2 Hz spike and wave discharges 5) Focal spikes and spike wave complexes

In addition to the characteristic electrographic bursts of abnormal activity that are recorded when epileptic patients experience a seizure (ictal episode), the electroencephalogram (EEG) of epileptics will normally display isolated sharp transients or "spikes" in some locations of the 
brain. These interictal spikes are a complementary source of information in the diagnosis and localization of epilepsy.

\section{Literature Reviews}

Beginning in the 1970s, Gotman began publishing papers on the automatic analysis of EEG waveforms. In 1983, he looked at coherence to measure interchannel differences in onset times to study seizure propagation [4]. A consequence of this work was an analysis technique for automated seizure detection.

Most recently, Zijlmans and Gotman have found that changes in parasympathetic activity (e.g., heart rate) frequently manifest prior to the earliest EEG evidence of seizure onset [5]. This is consistent with much earlier work qualitatively examining seizure-related heart rate changes (e.g., [6]).

Time-frequency methods for seizure detection have a long research history. In 1993, Zaveri et al. showed that the mean Teager energy of ECoG recordings yielded 100\% detection accuracy on an 11-seizure database [7]. In 1994 Schiff et al. They used wavelet transforms in the analysis of EEG signal. They compared the quality of feature extraction of continuous wavelet transforms using standard numerical techniques, with more rapid algorithms utilizing both polynomial splines and multiresolution frameworks. They further contrasted the difference between filtering with and without the use of surrogate data to model background noise, demonstrated the preservation of feature extraction with critical versus redundant sampling, and performed the analysis with wavelets using different shapes. They reported a dramatic reduction in computational time required to perform this analysis, without compromising the accuracy of feature extraction [8]. Williams and Zaveri continued their time-frequency analysis in 1995 and introduced there reduction interference distribution (RID) for seizure analysis [9].

They noted the presence of preseizure chirps, but did not present specific detection results. Similar publications in the same year reported chirp identification using scalograms and spectrograms [10], In 1995 Barreto et al. applied A wavelet transformation to electrocorticogram (ECoG) recorded from epileptic patients. The temporal sharpness associated with interictal spikes at different resolutions is observed and two ways for representing the multiresolution sharpness of the spikes are proposed. They found that a wavelet transformation is capable of separating a time series, such as the ECoG from an epileptic patient, according to the sharpness of the signal at different temporal resolutions. They also observed that interictal spikes display significant sharpness at several resolution levels [11]. In 2002, szilagyi et al. they found a method to detect epileptic in to two steps, first a multi-resolution step is applied to obtain the magnitudes of the different spectral components. These values then applied to the inputs of the artificial neural network (ANN), which gave the probability of the presence of the epileptic seizures. They found that the accuracy of the detection method was over 95\% [12]. In 2003 Chiri Yamaguchi, used wavelet transform to detect the local low frequency components in each normal and epileptic EEG. In his work, human normal and epileptic electroencephalogram (EEG) signals have been analyzed using Fourier Transform (FT) and Continuous Wavelet Transform (CWT) as well as Discrete Wavelet Transform (DWT). Using the power spectrum densities of both normal and epileptic EEG showed 1/f fluctuations as a function of frequency $f$. The alpha rhythms that appeared in the normal EEG were not observed in the epileptic EEG. FT couldn't reveal the localized spikes and complexes that are typical among epileptic seizures. On the contrary, both CWT and DWT analyses could clearly detect localized low-frequency epileptic components in the EEG records. He aimed in his study to find automatic detection of the epileptic disorders in the EEG in order to support the diagnosis and care of the epileptic syndromes and related seizure disorders. He proposed that wavelet transform solved both of these problems by replacing modulation with scaling to achieve frequency 1ocalization. Furthermore, the wavelet transform has 3 distinctive features: Multiresolution; Constant relative bandwidth, that is, the time-widths of the wavelets is adapted to their frequency; and Wavelets has the ability to indicate signal which is localized in time domain or frequency domain. It is called a mathematical microscope for analyzing signals [13]. In 2003, Arnaud Delorme and Scott Makeig analyzed 15 sessions of 64-channel electroencephalographic (EEG) data recorded from a highly trained subject during sessions in which they attempted to regulate power at $12 \mathrm{~Hz}$ over his left- and right-central scalp to control the altitude of a cursor moving toward target boxes placed at the top-, middle-, or bottomright of a computer screen. They used independent component analysis (ICA) to decompose 64-channel EEG data. They found clearly while this highly trained subject regulated the amplitude of his $12-\mathrm{Hz}$ EEG activity on the left- and right-central scalp, 
they simultaneously modulated his EEG dynamics at several cortical areas and frequencies. Therefore, learned modulation of one EEG amplitude measure may be effected through a process that concurrently modulates multiple EEG processes, producing event-related changes in power in multiple EEG components and frequencies [14].

In 2004, Quian, et al. present a method that combines the wavelet transform, which localizes distinctive spike features, with superparamagnetic clustering (SPC), which allows automatic classification of the data without assumptions such as low variance or Gaussian distributions. Moreover, an improved method for setting amplitude thresholds for spike detection is proposed. Supervised spike sorting of a large number of channels is highly time-consuming and nearly impossible to perform during the course of an experiment.

The conventional basic algorithmic steps of their spike classification are as follows:

Spike detection, spikes are detected with an automatic amplitude threshold on the high-pass filtered data. Extraction of distinctive features from the spike shapes, and Clustering of the spikes by these features. A small set of wavelet coefficients from each spike are chosen as input for the clustering algorithm. The SPC classifies the spikes according to the selected set of wavelet coefficients. An alternative approach is to define spike classes by a set of manually selected thresholds (window discriminators) or with spike templates. The procedure is fully unsupervised and fast, thus can be used for the classification of spikes from a large number of channels recorded simultaneously. Spike detection was achieved by using an amplitude threshold on the high-pass filtered data. The threshold value was calculated automatically using the median of the absolute value of the signal. The validity of the results is based on the shape and variance of the spike shapes, the interspike interval distribution, the presence of a refractory period, and so forth [15]. In 2004 Andrew B. Gardner used a method consisted of 12 patients to diagnose with medically resistant temporal lobe epilepsy during evaluation for epilepsy surgery. These patients collectively had 118 seizures during the recording period. The performance of the detection framework was assessed with an emphasis on four key metrics of seizure detection:

1. Sensitivity (probability of correct detection), 2. Mean detection latency, 3. Earlydetection fraction (prediction or detection of seizure prior to electrographic onset), and

4. False positive rate.
In particular, the online detector achieved $97.85 \%$ sensitivity with an average of 1.74 false positive predictions per hour (Fph). According to their results they claimed that their proposed detection approach is not only feasible for seizure analysis, but it is robust technique for detecting these events and provides a method for predicting $40 \%$ of seizures [16].

Other approaches for detection are also available in the literature. In 1990, Gottman reported results for 293 seizure recordings from 49 patients [17]. The reported average false alarm rate was one false positive per hour (Fph); $24 \%$ of all seizures went undetected. The false detection problem was then attacked by training a classifier to recognize false alarms.

In 1993, Gotman reported that the false detection rate for this method fell 33\% [18]. By 1995 , an early seizure warning system, trained on template EEG activity, was presented along with impressive detection results: $100 \%$ accuracy with an average latency of 9.35 seconds and a false alarm rate of $0.02 \mathrm{Fph} \mathrm{[19].} \mathrm{A} \mathrm{later} \mathrm{publication} \mathrm{[20]}$ reported similar results (9.35 second latency) using time- and frequency domain features classified by a k-nearest neighbor classifier. A total of 47 seizures from 12 patients were considered.

In 1997, Marchesi et al. explored genetic programming for learning to detect spike and-slowwave activity in EEG recordings using a variety of simple features [21]. No validation was performed on seizure data. In 1998, Osorio et al. achieved according to their claim a perfect detection using a measure called seizure intensity. The average detection latency was 2.1 seconds evaluated on a database of 125 patients, but the same data was used for training and validation [22]. In 2001, they qualitatively described a system for real-time detection and intervention with direct electrical stimulation of the brain [23]. More recent seizure intensity results using offline $\mathrm{ECoG}$ recordings were reported in 2002 [24]. In 2004, Xiaoli et al. proposed a recurrence quantification analysis (RQA) to describe dynamical characteristics of EEG recordings on rat experiments, which is helpful to predict seizures. A series of experimental tests in this study show that the dynamical characteristics of EEG data with RQA can identify the differences among inter-ictal, pre-ictal and ictal phases; RQA measures over time, a movingwindow technique is applied to a long-term rat EEG data. EEG signal firstly is divided into segments of 5 seconds (1000 points), and then the Recurrence Plots (RP) is computed after embedding the EEG segment. 
Finally, three RQA measures are calculated to discover the EEG dynamics over time. The interictal phase, which indicated that the brain electrical activity share a similar underlying dynamic in this period. During the extended pre-ictal phase, \%REC decreases to a minimum value prior to the onset of the seizures. However, an altered state of the brain dynamics is hard to be observable from EEG time series itself. \%DET are sensitive to quickly and highly the fluctuating EEG from the observation of recurrence rate. It is found that the three measures \%REC, determinism \%DET, and entropy ENTR over the entire EEG recordings can track the complexity changes of brain electrical activity [25]. In 2004, Adjouadi et al. used the Walsh transformation to detect interictal spikes in electroencephalogram (EEG) data. Independent sets of EEG data recorded on 18 patients with focal epilepsy were used to train and test the algorithm. Twenty to thirty minutes of recordings were obtained with each subject awake, supine, and at rest. Spikes were annotated independently by two EEG experts. On evaluation, the algorithm identified 110 out of 139 spikes identified by either expert (True Positives $=79 \%$ ) and missed 29 spikes (False Negatives $=21 \%$ ). Evaluation of the algorithm revealed a Precision (Positive Predictive Value) of $85 \%$ and a Sensitivity of $79 \%$. The encouraging preliminary results support its further development for prolonged EEG recordings in ambulatory subjects. With these results, the false detection (FD) rate is estimated at 7.2 FD per hour of continuous EEG recording [26].

R. J. Povinelli, et al. [27] Firstly construct the phase space using an appropriate time lag which is calculated using the first minimum of the automutual information function, the reconstructed phase space (RPS) dimension is calculated using the global false nearest-neighbor technique, then build Gaussian mixture models(GMM) for each signal class from the reconstructed phase space. The classification method was done with a maximum likelihood Bayes classifier. This technique is applied to three data sets; motor current, ECG and TIMIT speech corpus. In ECG application four different types of rhythms are classified; SR, MVT, PVT and VF.

R.J.Povinelli, et al. [28] used distribution models as statistical representations over multidimensional reconstructed phase space both nonparametric distributions based on binning and occurrence counts and parametric distributions based on Gaussian Mixture Model (GMM). They used classification methods such as Bayes maximum likelihood and ANN. This paper applied its technique on heart arrhythmias and speech recognition. A phase space is constructed by using delayed versions of the original signal; by selecting the appropriate dimension time lag of the RPS to give the maximum classification accuracy. For arrhythmias classification the best accuracy was 95.5\% by using GMM model and Bayes classifier, and $62.6 \%$ for speech recognition.

Report of the American Academy of Neurology and the American Clinical Neurophysiology Society [29] compared the normative values uses group statistics to determine whether a parameter (or parameters) measured on an individual patient lies inside or outside the range of normal values, and statistical techniques employed may be simple thresholds based on the mean and standard deviation of a "normal" distribution. The report said that diagnostic discriminant analysis gathers selected parameters for several different patient diagnostic subgroups, as well as for controls. A discriminant function can be mathematically determined that ascribes certain patterns of these parameters to each patient group. The technique then compares the pattern of the EEG parameters derived from one patient to all of the relevant patient groups to determine with which diagnostic group the patient's EEG is statistically most closely associated.

It found that digital spike and seizure detection can identify candidate events that might be epileptic spikes or seizures, although frequent false-positive detections occurred. The clinical use of any spike or seizure-detection algorithm must balance sensitivity against specificity. Controlled studies compared digital detection to detection by visual review as the standard. Sensitivities were often better than 80 to $90 \%$, although specificity remained poor. The clinical rationale seems clear.

Other approaches for detection have included: autoregressive (AR) modeling, rulesbased analysis of amplitude and frequency changes of scalp EEG singularspectrum techniques for scalp EEG, and nonlinear techniques like multidimensional probability evolution.

\section{Methodology}

Mathematical transformations are applied to signals to obtain further information from that signal that is not readily distinguishable in the raw signal. Most of the signals in practice are timedomain signals in their raw format. That is, whatever that signal is measuring, is a function of time. For example, looking at an EEG signal (Electroencephalogram, graphical recording of brain electrical activity), the typical shape of a healthy 
EEG signal is well known to neurophysiologist. Any significant deviation from that shape is usually considered to be a symptom of a pathological condition. This pathological condition, however, may not always be quite obvious in the original time-domain signal. Neurophysiologists usually use the time-domain EEG signals to analyze EEG. This, of course, is only one simple example why frequency content might be useful.

Wavelet analysis represents the next logical step: a windowing technique with variable-sized regions. Wavelet analysis allows the use of long time intervals where we want more precise lowfrequency information, and shorter regions where we want high-frequency information. To make wavelet transformation, the time-domain signal from various high pass and low pass filters, which filters out either high frequency or low frequency portions of the signal. This procedure is repeated, every time some portion of the signal corresponding to some frequencies being removed from the signal [30,32]. The DWT is considerably easier to implement when compared to the CWT. The basic concepts of the DWT will be introduced in this section along with its properties and the algorithms used to compute it. In the discrete case, filters of different cutoff frequencies are used to analyze the signal at different scales. The signal is passed through a series of high pass filters to analyze the high frequencies, and it is passed through a series of low pass filters to analyze the low frequencies.

Calculating wavelet coefficients at every possible scale is a fair amount of work, and it generates a lot of data. Then the analysis will be much more efficient and just as accurate, then discrete wavelet transform (DWT) is obtained $[30,32,33]$.

Given a signal $\mathrm{S}$ of length $\mathrm{N}$, the DWT consists of $\log 2 \mathrm{~N}$ stages at most. Starting from S, the first step produces two sets of coefficients: approximation coefficients cal, and detail coefficients cd1. These vectors are obtained by convolving $\mathrm{S}$ with the low-pass filter Lo_D for approximation, and with the high-pass filter Hi_D for detail, followed by dyadic decimation. The length of each filter is equal to $2 \mathrm{~N}$. If $\mathrm{n}=$ length $(\mathrm{S})$, the signals $\mathrm{F}$ and $\mathrm{G}$ are of length $\mathrm{n}+2 \mathrm{~N}-1$, and then the coefficients cal and cd1 are of length is (n1) $/ 2+N$

The next step splits the approximation coefficients cal in two parts using the same scheme, replacing $\mathrm{S}$ by cal and producing $\mathrm{ca} 2$ and $\mathrm{cd} 2$, and so on.

In general case the decomposition step for one-dimensional DWT is in the block diagram shown below. It can be seen that for level $\mathrm{J}+1$ the input signal $\mathrm{S}=\mathrm{ca} \mathrm{J}$, then after low pass and high pass filtering the signal is down sampling by 2 to produce ca $\mathrm{J}+1$ at the output, So the wavelet decomposition of the signal $\mathrm{S}$ analyzed at level $\mathrm{j}$ has the following structure: [caj, cdj, ..., cd1].

The standard deviations are calculated for each coefficient selected above. Where the standard deviation is

$$
S T D=\sqrt{\frac{1}{n-1} \sum_{i=1}^{n}\left(x_{i}-\mu\right)^{2}}
$$

Power spectral density (PSD) refers to the amount of power per unit (density) of frequency as a function of the frequency. The power spectral density of each wavelet coefficient ca1, ca2, and ca3 is calculated.

Another factor is used in this paper is entropy. The entropy of signal is a measure of the randomness of the signal. The entropy of each beat was first calculated with two commonly used types of the entropy, Shannon entropy and log-energy entropy.

The used methods are the Independent Component Analysis, Artificial Neural Networks, Support Vector Machines, Decision Trees, and Naïve Bayes, which will be used later in the experimental part.

Independent Component Analysis transform the data $\mathrm{x}$ into maximally independent variables. This method was proposed by Jeanny Hérault and Bernard Ans. Basically, this method uses the equation $\mathrm{s}=\mathrm{Wx}$, whereas $\mathrm{W}$ is linear static transformation, $\mathrm{x}$ is the original data, and $\mathrm{s}$ is the hidden components.

Artifical Neural Network adopts the concept of biological neural networks. The artificial neural network is composed of a set of connected artificial neurons. Each connection has a weight, and the output of each neuron is a nonlinear function. The network is composed of three main layers: input, hidden, and output layers. The weights adjust according to the training data prediction error. The final weights are used in the classification of testing records. Recently, the number of hidden layers was increased, which introduced the deep learning topic. This improvement in the structure of the network contributed in increasing the accuracy of the classifier. Deep learning requires large amount of data to provide the optimal performance.

Naïve Bayse Classifier is a classification algorithm that relies on conditional probability. It utilizes Bayes' theorem and assumes a strong 
independence between features. It computes the occurrences of the features and the relation between them in the corpus considered. NB is good in practice even when the dimensionality of the input is high. NB is a good classifier for TC in general.

Decision Trees uses the training data to build a tree model that can be used later for classification purposes. Currently, many decision tree algorithms exist including Random Forest, Random Tree, J48, and CART.

\section{Experiments and Results}

This chapter describes the experimental work that was performed to distinguish between normal and abnormal EEG signals. The first section of this chapter introduces the data utilized in this research and it is characteristics. The second section describes the first technique, which is the Wavelet transform of the EEG signals. The third section describes the second technique used in this work, which consists of Wavelet Transform and the phase space for the wavelet coefficients. The fourth section provides another analysis technique that depends only on the Phase Space plots without any transformation. The fifth section employs another analysis technique which is based on Fourier analysis only. The final section of this chapter provides a comparison among the different used techniques and their counter parts in the literature and discussion for all considered techniques.

\subsection{Data}

The datasets used in this research are selected from the Epilepsy center in Bonn, Germany by Ralph Andrzejak [41].

Five data sets containing quasi-stationary, artifact, e.g., due to muscle activity or eye movements, free EEG signals both in normal subjects and epileptic patients. Two sets denoted A, $\mathrm{E}$ are used in this work. Each set contains 100 single channel EEG segments of 23.6-sec duration. These segments were selected and cut out from continuous multichannel EEG recordings. Set A consisted of segments taken from surface EEG recordings that were obtained from five healthy volunteers using a standardized electrode placement. Volunteers were relaxed in an awake state with eyes open. Set E only contained seizure activity. All EEG signals were recorded with the same 128-channel amplifier system, using an average common reference (omitting electrodes containing pathological activity) E or strong eye movement artifacts. After 12 bit analog-to-digital conversion, the data were written continuously onto the disk of a data acquisition computer system at a sampling rate of fs $=173.61 \mathrm{~Hz}$. [40]. In all techniques 20 normal and abnormal single channel EEG segments are selected.

\subsection{Experiments}

Discrete wavelet transform DWT is used to decompose the EEG signal of each band of coefficients of normal and abnormal signals. DWT is taken of EEG signal for 3 levels using daubchies wavelet based function $\mathrm{db} 4$ that is the most common because of it is favorable characteristics, such as orthogonal and filters length that can be determined as the work needed. To determine the coefficients of each signal, wavelet coefficients ca1, $\mathrm{ca} 2$, and ca3 with frequency ranges of 0 -fs $/ / 2,0$ fs $/ 4,0$-fs $/ 8$, respectively are selected. These ranges contain the frequencies of EEG rhythms alpha, beta, theta, and delta. After that the statistical classification and power spectral density are taken to make classification of EEG signals.

The values of the standard deviation of each coefficient of normal and abnormal signals are calculated then power spectral density will be calculated.

Figures 1-6 display the power spectral density of each wavelet coefficient ca1, ca2, and ca3. The figures of the abnormal case show that the spikes appear in the range of $1-4 \mathrm{~Hz}$ with maximum power (maximum amplitudes), but they don't appear in normal.

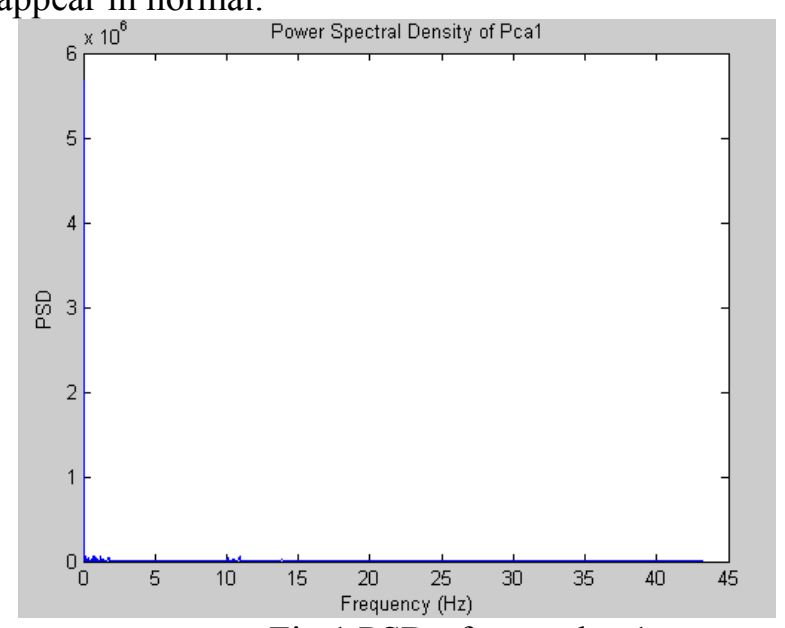

Fig.1 PSD of normal cal 


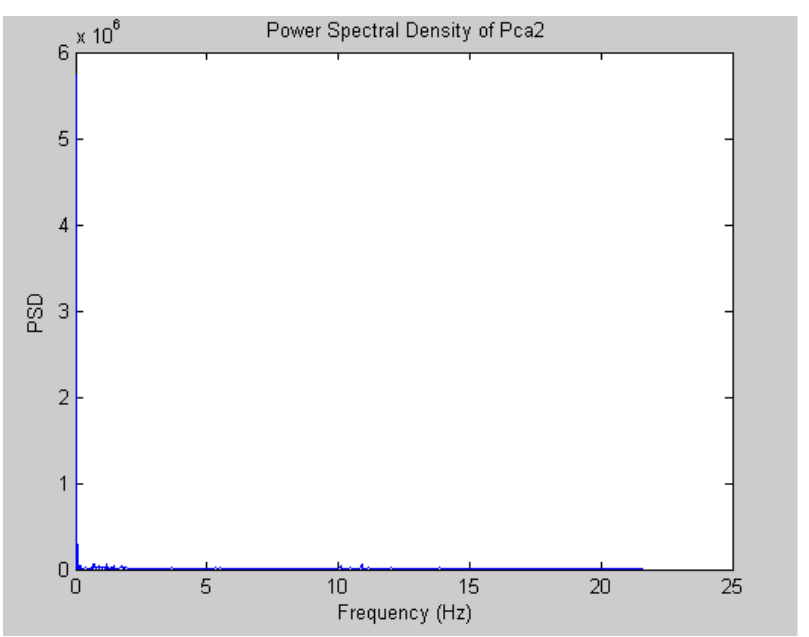

Fig.2 PSD of normal ca2

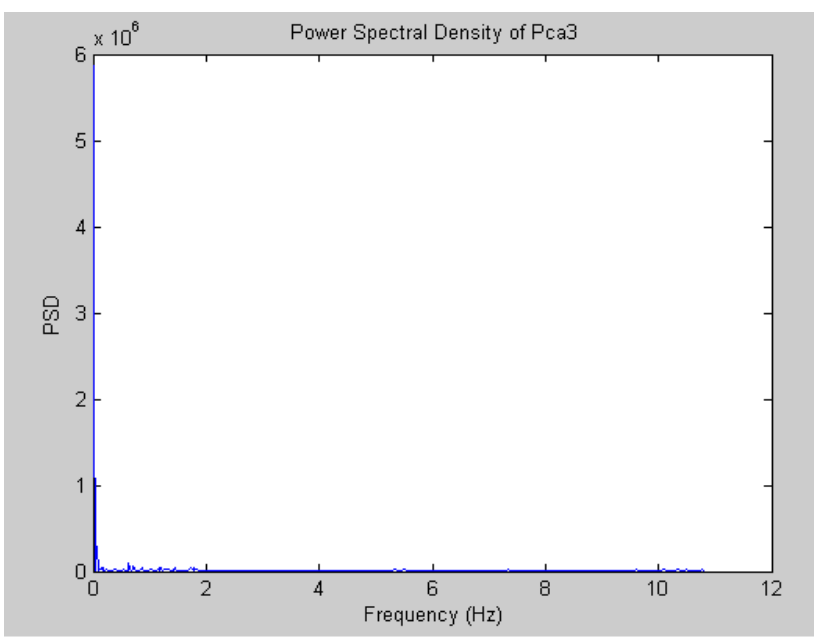

Fig.3 PSD of normal ca3

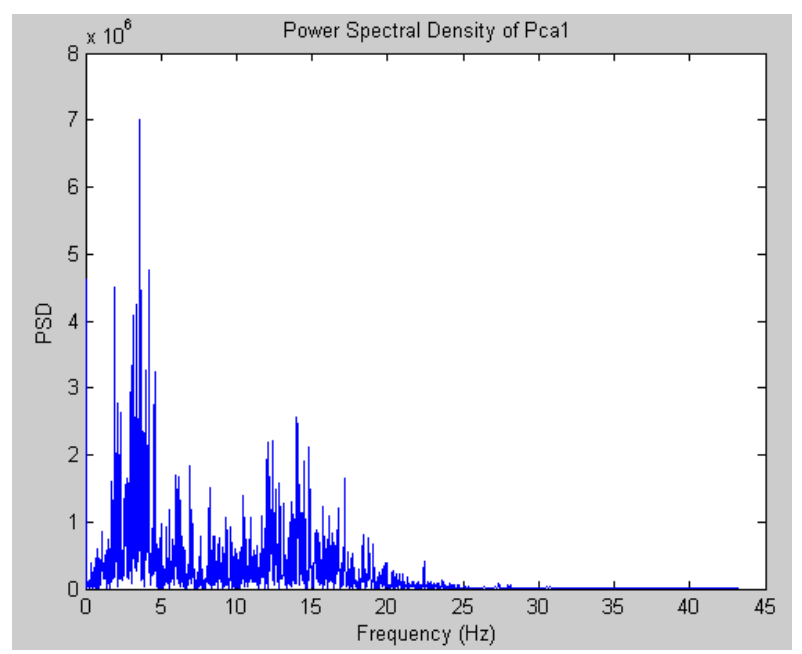

Fig.4 PSD of abnormal cal

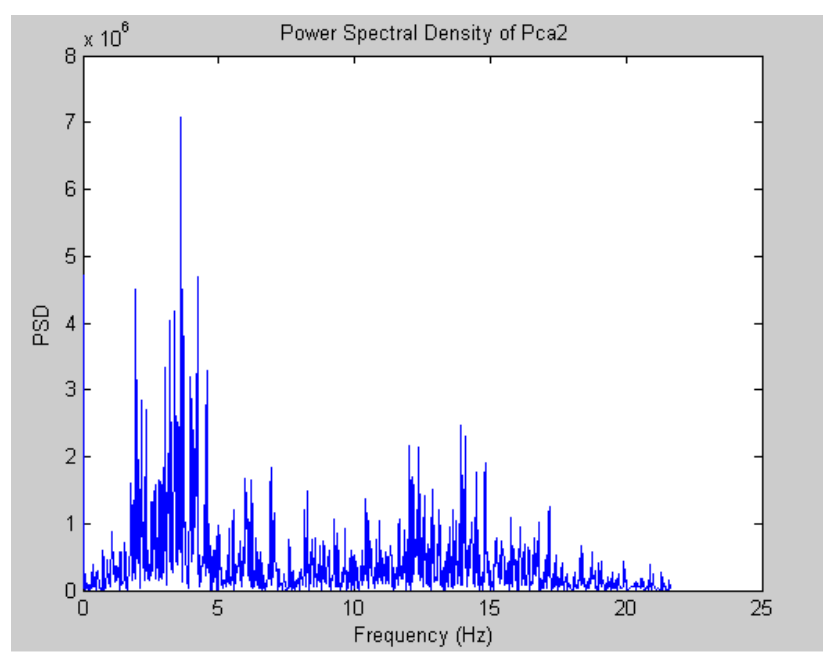

Fig.5 PSD of abnormal ca2

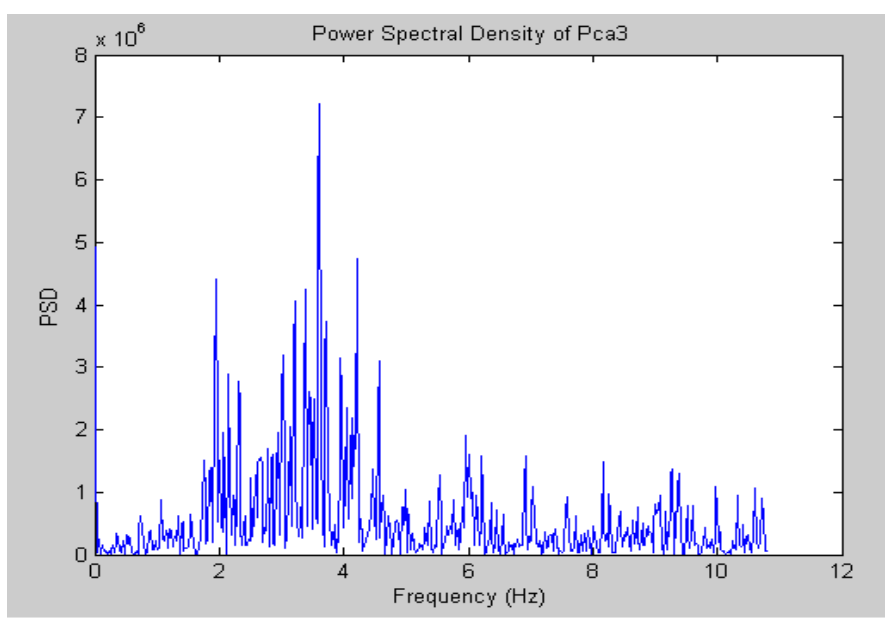

Fig.6 PSD of abnormal ca3

The total number of signals used are 150 signals. As for the classifiers, we used five classifiers from the literature. Namely, we compared SVM, ANN, Naïve Bayes, and Decision Trees. As for the implementation of these classifiers, we used WEKA toolkit.

Regarding the evaluation measurements, we used training time, classification time, and F1 measurement, they are defined as follows.

- Training time: It is the time needed to train the classifier and build the model.

- Testing time: It is the time needed to classify the testing instances.

- F1 Measurement: It is an indication of the classification accuracy. It is defined as the harmonic mean of the recall and the precision. Its formula is given as follows. 


$$
\mathrm{F}_{1}=\frac{2 * R * P}{R+P},
$$

Whereas $\mathrm{R}$ presents the recall, which is the percentage of retrieved relevant and $\mathrm{P}$ presents the precision. The formulas of recall and precision are given as follows.

$$
\begin{aligned}
& \mathrm{R}=\frac{T P}{T P+F \text { 湩 }} \\
& \mathrm{P}=\frac{T P}{T P+F P},
\end{aligned}
$$

Whereas TP is true positives, FP is the false positive From Table 1, it is obvious that the use of ICA proved its high performance in texture classification. This is reasonable as ICA is capable of eliminating noise from images and concentrating on important features only. Furthermore, Artificial neural network classifier proved to be of superior accuracy, but its training and testing time is slightly larger than other classifiers. On the other hand, Naïve Bayes proved to be a very fast classifier that works efficiently with big datasets, despite its average accuracy compared with other classifiers. Therefore, the choice of the classifier is domain dependent, as certain domains seek the optimal accuracy while others seek fast execution time.

Table 1 Performance Measurement

\begin{tabular}{|l|l|l|l|}
\hline Classifier & $\begin{array}{c}\text { Training } \\
\text { Time }\end{array}$ & $\begin{array}{l}\text { Classification } \\
\text { Time }\end{array}$ & $\begin{array}{l}\text { F1 } \\
\text { Measurement }\end{array}$ \\
\hline SVM & 1.05 & 10 & 0.705 \\
\hline $\begin{array}{c}\text { Artificial } \\
\text { Neural } \\
\text { Networks }\end{array}$ & 26.6 & 240 & $\mathbf{0 . 9 0 9}$ \\
\hline $\begin{array}{c}\text { Decision Trees } \\
\text { (J48) }\end{array}$ & 0.39 & $\mathbf{1}$ & 0.76 \\
\hline \begin{tabular}{c} 
Naïve Bayes \\
\hline
\end{tabular} & $\mathbf{0 . 0 3}$ & $\mathbf{1}$ & 0.837 \\
\hline
\end{tabular}

\section{Discussion}

Wavelet transform is used to decompose the EEG signal as seen in the first technique. Discrete wavelet transform applied for EEG signal for 3 levels to determine the coefficients of the signals, this helps in limiting the bands of the frequencies that contain spikes in the signal, Then standard deviation of the coefficients of normal and abnormal signals are calculated where the standard deviation of abnormal signal is higher than the normal case. The power spectral density of each band is plotted. From the plots frequency range of the spikes were detected to be from 1-4 Hz. In this technique the frequency bands are determined and the spikes caused by epileptic seizures can be detected. In this technique the overall accuracy is $100 \%$.

It can be noticed that using wavelet transform in the first technique allows extracting low and high frequencies of the EEG signal, after that the standard deviation parameter used can classify the signals to determine the abnormality with overall accuracy $100 \%$. The power spectrum of wavelet based coefficients proved that the spikes happened in the lower frequencies in the delta range (i.e. 1-4 Hz). This technique used simple parameters to detect epileptic seizures and determine its frequency content, while using phase space reconstruction only detects the disorder epileptic seizures considering the deviation between the amplitudes of normal and abnormal signals with an overall accuracy of $90 \%$.

From the above it can be seen that using wavelet transformation will improved the accuracy of the technique, this is because wavelet transform is a multiresolution, it gives good resolution in time domain and good resolution in frequency domain, in wavelet transform the width of the window used in transformation is changed (i.e. in time) as the transform is computed for every single spectral component (i.e. in frequency), while reconstructed phase space is time domain representation only; on the other hand Fourier transform is frequency domain representation only.

\section{Conclusions}

This research presents a framework for analyzing EEG time-series data and demonstrates its use for a practical problem: detecting seizures from the intracranial EEG (seizure analysis). The methodology of this research used a modified technique in analyzing EEG signal to distinguish between normal and abnormal (epileptic seizures). The data used in this research is long and it is taken from multichannels of the brain. The technique consists of wavelet transform, this technique showed very high accuracy reached to $100 \%$, in this technique it can be easily to detect disorder in the brain using simple classifier depends on statistical calculation which is the standard deviation STD, the technique detected the spikes caused by epilepsy and determined easily their locations (i.e. frequency ranges) using simple approach that is the power spectral density of the wavelet based coefficients. The wavelet transform used in this technique has many advantages over other transforms, like Fourier 
transform, such that it can preserve both frequency and timing information and it can simultaneously extract both low frequency and high frequency signal with different frequency resolutions.

\section{References}

[1] www.bEEG-MEG

[2] Szilagyi L., Z.benyo, S.M. Szilagyi "A new method for epileptic waveform recognition using wavelet decomposition and artificial neural Networks", IEEE trans. Houston, TX, USA, October 2002 .

[3] Barreto A., N. Chin., J. Andrian J. Riley " Multiresolution Characterization of Interictal Epileptic Spikes based on a Wavelet Transformation". University Park, Miami, Florida, 33199

[4] Gotman .J, 1985 "Seizure recognition and analysis," Electroencephalogram Clinical Neurophysiology Suppl,vol.37, pp. 133-45,

[5] Zijlmans. M, Flanagan .D, and Gotman .J, 2002,"Heart rate changes and ECoG abnormalities during epileptic seizures: prevalence and definition an objective clinical sign," Epilepsia, vol. 43, pp. 847-54,

[6] Theodore .H and Haas .L, 1991, "A personal computer controlled long-term video-EEG monitoring, seizure detection, and collection system,"American Journal of EEG Technology, vol. 31, pp. 103-108,

[7] Zaveri .H, Williams .W, and Sackellares .J, 1993, "Energy based detection of seizures", presented at 15th Annual International Conference on Engineering and Medicine in Biology.

[8] Schiff .J, Aldroubi .A, Unser .M, and Sato .S, 1994, " Fast Wavelet Transformation of EEG Electroencephalography and Clinical Neurophysiology", 91, 442 .

[9] Williams .W, Zaveri .H, and Sackellares .J, 1995 "Time-Frequency Analysis of Electrophysiology Signals in Epilepsy," IEEE Engineering in Medicine and Biology, pp. 133143 ,

[10] Benedetto .J and Colella .D, 1995. "Wavelet analysis of spectrogram seizure chirps,"presented at Wavelet Applications in Signal and Image Processing III",

[11] Barreto .A, Chin .N, Andrian .J. Riley .J, " Multiresolution Characterization of Interictal Epileptic Spikes based on a Wavelet Transformation". University Park, Miami, Florida, 33199.
[12] szilagyi .L, benyo .Z, Szilagyi .S, 2002 "A new method for epileptic waveform recognition using wavelet decomposition and artificial neural networks", IEEE trans. Houston, TX, USA,

[13] Chiri Yamaguchi, 2003, "Fourier and Wavelet Analyses of Normal and Epileptic

Electroencephalogram (EEG)" Proceedings of the 1st International IEEE EMBS Conference on Neural Engineering Capri Island, Italy.

[14] Arnaud Delorme and Scott Makeig, 2003 "EEG Changes Accompanying

Learned Regulation of 12-Hz EEG Activity", IEEE, VOL. 11, NO. 2,

[15] Quiroga .R, Nadasdy .Z Ben-Shaul .Y, 2004 LETTER Communicated by Maneesh Sahani "Unsupervised Spike Detection and Sorting with Wavelets Superparamagnetic Clustering Neural Computation" 16, 1661- 1687 Massachusetts Institute of Technology.

[16] Gardner .A, 2004"A Novelty Detection Approach to Seizure Analysis from Intracranial EEG". A Dissertation Presented in Doctor of Philosophy to the academic faculty in Electrical Engineering School of Electrical and Computer Engineering "Georgia Institute of Technology" Atlanta, GA USA

[17] Gotman .J, "Automatic seizure detection: improvements and evaluation,"

Electroencephalogr Clin Neurophysiol, vol. 76, pp. 317-24, 1990.

[18] Qu .H and Gotman .J, 1993, "Improvement in seizure detection performance by automatic adaptation to the EEG of each patient," Electroencephalogr Clin Neurophysiol, vol. 86, pp. 79-87,

[19] Qu .H and Gotman .J, 1995, "A seizure warning system for long-term

epilepsy monitoring," Neurology, vol. 45, pp. 2250-4,

[20] Qu .H and Gotman .J, 1997,"A patient-specific algorithm for the detection of seizure onset in long-term EEG monitoring: possible use as a warning device," IEEE Trans Biomed Eng, vol. 44, pp. 115-22,

[21] Marchesi .B, Stelle .A, and Lopes .H, 1997 "Detection of epileptic events using genetic programming," presented at Proceedings of the 19th Annual International Conference of the IEEE Engineering in Medicine and Biology Society, Chicago, IL, USA,

[22] Osorio .I, Frei .M, and Wilkinson .S, 1998,"Real-time automated detection and

quantitative analysis of seizures and short-term prediction of clinical onset," 
Epilepsia, vol. 39, pp. 615-27,

[23] Osorio .I, Frei .M, Manly .B, Sunderam .S, Bhavaraju .N, and Wilkinson .S, 2001, "An introduction to contingent (closed-loop) brain electrical stimulation for seizure blockage, to ultra-short-term clinical trials, and to multidimensional statistical analysis of therapeutic efficacy," J Clin Neurophysiol, vol. 18 , pp. 533-44,

[24] Osorio .I, Frei .M, Giftakis .J, Peters .T, Ingram .J, Turnbull .M, Herzog .M,

2002,"Performance reassessment of a real-time seizure-detection algorithm on long ECoG series," Epilepsia, vol. 43, pp. 1522-35,

[25] Xiaoli Li,.., Gaoxiang Ouyang , Xin Yao , Xinping Guan , 2004 "Dynamical characteristics of pre-epileptic seizures in rats with recurrence quantification analysis" Elsevier B.V. All rights reserved. doi:10.1016/j. physleta.

[26] Adjouadi .M, Sanchez .D, Cabrerizo .M, Ayala .M, Jayakar .P, Yaylali .I, and Barreto .A, 2004, "Interictal Spike Detection Using the Walsh Transform"

IEEE Transactions on Biomedical Engineering, Vol. 51 , No.

[27] Povinelli R.J., Johnson M.T., Lindgren A.C. ,Roberts F.M. and Ye J., "Statistical Models of Reconstructed Phase Space for signal classification", IEEE Transactions on Signal Processing(in press).

[28] Povinelli R.J., Johnson M.T., Lindgren A.C. and Ye J.2004, "Time series classification using Gaussian mixture models of reconstructed phase spaces" IEEE Transactions On Knowledge And Data Engineering, VOL. 16, NO. 6

[29] Report of the American Academy of Neurology and the American Clinical Neurophysiology Society

[30] www.users.rowan.edu/ polikar

[31] www.astronomy.swin.edu.au

[32] Matlab Help Wavelet toolbox from Matlab Manual comments

[33] Class notes university of acron 2003 "wavelet for engineering and science"

[34] Martinerei J., C. Adam. Le van Quyen, M. Baul , S. Clemenceau, B. Renault \& F.J. Varela" Epileptic seizures can be anticipated by non-linear analysis". NATURE MEDICINE • VOL $4 \cdot 1998$

[35] [Book] Kantz H. and Schrreiber T.1997, "Non Linear time series analysis". Cambridge: Cambridge University Press.
[36]: Boker S.M., 1998," Linear and Nonlinear Dynamical Systems Data Analytic Techniques and an Application to Developmental Data" $\mathrm{PhD}$ thesis, Department of Psychology, University of Virginia, USA.

[37] Cao L.1997, "Practical method for determining the minimum embedding dimension of a scalar time series", Physica D, vol 110, Pp. 43-50

[38] Strogaz S.H.1994, "Nonlinear Dynamics and Chaos: with application to physics, biology, chemistry", and engineering, Addison-Wesley Publishing co., MA, USA.

[39] 'Indications of nonlinear deterministic and finite-dimensional structures in time series of brain electrical activity: Dependence on recording region and brain state' Physical Review, Vol. 64, 0619072001 The American Physical Society

[40] Andrzejak G.,Klaus Lehnertz, Florian Mormann, Christoph Rieke, Peter David,and Christian E. Elger1

[41]http://www.meb.unibonn.de/epileptologie/scien ce/physik/eegdata.html 